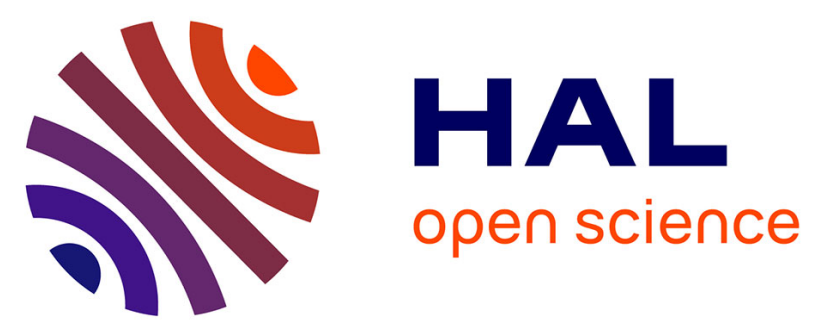

\title{
A Metrological Measurement of Texture in Hyperspectral Images Using Relocated Spectral Difference Occurrence Matrix
}

Rui Jian Chu, Noël Richard, Christine Fernandez-Maloigne, Jon Yngve Hardeberg

\section{To cite this version:}

Rui Jian Chu, Noël Richard, Christine Fernandez-Maloigne, Jon Yngve Hardeberg. A Metrological Measurement of Texture in Hyperspectral Images Using Relocated Spectral Difference Occurrence Matrix. 2019 IEEE International Conference on Image Processing (ICIP), Sep 2019, Taipei, Taiwan. pp.3133-3137, 10.1109/ICIP.2019.8803378 . hal-02481586

\author{
HAL Id: hal-02481586 \\ https://hal.science/hal-02481586
}

Submitted on 21 Feb 2020

HAL is a multi-disciplinary open access archive for the deposit and dissemination of scientific research documents, whether they are published or not. The documents may come from teaching and research institutions in France or abroad, or from public or private research centers.
L'archive ouverte pluridisciplinaire HAL, est destinée au dépôt et à la diffusion de documents scientifiques de niveau recherche, publiés ou non, émanant des établissements d'enseignement et de recherche français ou étrangers, des laboratoires publics ou privés. 


\title{
A METROLOGICAL MEASUREMENT OF TEXTURE IN HYPERSPECTRAL IMAGES USING RELOCATED SPECTRAL DIFFERENCE OCCURRENCE MATRIX
}

\author{
Rui Jian Chu ${ }^{a}$, Noel Richard ${ }^{a}$, Christine Fernandez-Maloigne ${ }^{a}$, Jon Yngve Hardeberg ${ }^{b}$ * \\ ${ }^{a}$ XLIM Laboratory, JRU CNRS 7252, University of Poitiers, France \\ ${ }^{b}$ Department of Computer Science, Norwegian University of Science and Technology, Norway
}

\begin{abstract}
A new hyperspectral texture descriptor, Relocated Spectral Difference Occurrence Matrix (rSDOM) is proposed. It assesses the distribution of spectral difference in a given neighborhood. For metrological purposes, rSDOM employs Kullback-Leibler pseudo-divergence (KLPD) for spectral difference calculation. It is generic and adapted for any spectral range and number of band. As validation, a texture classification scheme based on nearest neighbor classifier is applied on HyTexiLa dataset using rSDOM. The performance is close to Opponent Band Local Binary Pattern (OBLBP) with classification accuracy of $94.7 \%$, but at a much-reduced feature size ( $0.24 \%$ of OBLBP's) and computational complexity.
\end{abstract}

Index Terms - hyperspectral, texture, non-uniformity, metrology, Kullback-Leibler

\section{INTRODUCTION}

Nowadays, the application of hyperspectral imaging (HSI) can be found everywhere. Ever since its deployment in remote sensing in the 1970's [1], HSI technology has been extended into many fields such as agriculture [2], medicine [3] and food quality inspection [4]. With the growing integration of HSI technology in our daily life, it becomes essential to develop adapted metrological solutions based on the spectral measurements. This is to ensure the reproducibility, accuracy and all the other expected properties when precise measurement are required for diagnosis, control or decision-making.

Texture or non-uniformity assessment was one of the first tasks developed in image processing. It originates from the psychophysical findings of Julesz which inspires the development of Haralick's texture features [5]. Since then, there has been a long list of texture feature propositions $[6,7,8]$. Nevertheless, texture evaluation for multivariate images (color, multi- and hyperspectral) is still an open question. The most direct solution is to perform assessment in a marginal way (band by band) or in a cross-channel processing $[9,10]$. Few texture features are developed specifically for hyperspectral

\footnotetext{
*This work is supported by the French national projects ANR DigiPi and ERDF NUMERIC /e-Patrimoine.
}

image processing such as 3D gray-level co-occurrence matrix [11] and three-dimensional wavelet texture feature [12].

Hyperspectral image processing is often faced with the curse of dimensionality due to the large number of spectral bands. As such, dimensionality reduction $[13,14]$ or band selection $[15,16]$ are usually performed prior to texture assessment. Such approaches are data-dependent as the result depends on the image content instead of the actually measured spectra. Hence, they are not adapted for metrological purposes as results from different dataset are incomparable.

The rest of the article is organized as follows. Section 2 recalls the definition of an electromagnetic spectrum and its similarity measure in a metrological context. Next, Section 3 details the hyperspectral texture descriptor. Section 4 then presents a texture classification scheme with analysis and discussion. Finally, Section 5 provides the concluding remarks.

\section{A METROLOGICAL CONSIDERATION}

Under the point of view of signal and image processing, a spectrum $S$ is defined as a continuous function $S=f(\lambda)$ over the wavelengths $\lambda$. Due to sampling operation, its hyperspectral acquisition is given by a discrete sequence of measurements $S=\left\{s(\lambda), \forall \lambda \in\left[\lambda_{\min }, \lambda_{\max }\right]\right\}$. This causes spectra to be considered as a set of independent measures, hence as vectors, probability density functions or sequences and associated with L2-norm for distance assessment.

The limits of such definition and of the use of L2-based metrics in the context of metrological processing have been shown in [17]. In order to obtain an adapted spectral difference respecting the metrological constraints, KullbackLeibler pseudo-divergence (KLPD) is introduced [18]. Considering two spectra $S_{1}$ and $S_{2}$, KLPD combines their shape difference $\Delta G$ and intensity difference $\Delta W$ as:

$$
d_{K L P D}\left(S_{1}, S_{2}\right)=\Delta G\left(S_{1}, S_{2}\right)+\Delta W\left(S_{1}, S_{2}\right)
$$

where:

$$
\begin{aligned}
& \Delta G\left(S_{1}, S_{2}\right)=k_{1} \cdot K L\left(\bar{S}_{1} \| \bar{S}_{2}\right)+k_{2} \cdot K L\left(\bar{S}_{2} \| \bar{S}_{1}\right) \\
& \Delta W\left(S_{1}, S_{2}\right)=\left(k_{1}-k_{2}\right) \log \left(\frac{k_{1}}{k_{2}}\right)
\end{aligned}
$$


noting that the normalized spectrum $\bar{S}$ is given by:

$$
\bar{S}=\left\{\bar{s}(\lambda)=\frac{s(\lambda)}{k}, \forall \lambda \in\left[\lambda_{\min }, \lambda_{\max }\right]\right\}
$$

with the normalization constant $k$ :

$$
k=\int_{\lambda_{\min }}^{\lambda_{\max }} s(\lambda) d \lambda
$$

and the KL divergence:

$$
K L\left(\bar{S}_{1} \| \bar{S}_{2}\right)=\int_{\lambda_{\min }}^{\lambda_{\max }} \bar{S}_{1}(\lambda) \cdot \log \frac{\bar{S}_{1}(\lambda)}{\bar{S}_{2}(\lambda)} d \lambda
$$

For a demonstration of KLPD application, consider the Food images from HyTexiLa hyperspectral texture dataset. We split each image into 25 patches from which an average spectrum is extracted a marginal way (Equation 10). Using KLPD, we calculate their spectral difference with respect to that of "Oregon". This is shown in Figure 1a which illustrates the average spectra ( 25 for each image) and Figure $1 \mathrm{~b}$ which plots the spectral shape and intensity differences.

\section{MEASURING HYPERSPECTRAL TEXTURE}

The first Julesz conjecture describes the pre-attentive discrimination of textures based on second-order statistics [19]. Under this context, we formulate a texture descriptor with reference to Haralick's texture features and Local Binary Pattern (LBP). To increase discrimination, we propose a joint spatialspectral formulation together with its similarity measure.

\subsection{Spectral Difference Occurrence Matrix}

The co-occurrence matrix in Haralick's [5] gives precise texture description, but the subsequent reduction into moments for similarity assessment leads to reduced efficiency. By contrast, LBP [8] provides weak texture characterization due to the binarization, but its similarity measure using Kullback discrimination is extremely efficient. Combining their strength and considering the relationship between co-occurrence and histogram of difference as defined by Unser [20], we introduce the Spectral Difference Occurrence Matrix (SDOM):

$$
\begin{gathered}
M^{(l, \theta)}(\Delta G, \Delta W)=\operatorname{Prob}\left(d_{K L P D}\left(S_{i}, S_{j}\right)=(\Delta G, \Delta W)\right), \\
\forall i, j \in I,\|\overrightarrow{i j}\|=l, \angle \overrightarrow{i j}=\theta
\end{gathered}
$$

which is defined over hyperspectral image $I$. It expresses the probability of finding a specific spectral difference (KLPD) $d_{K L P D}\left(S_{i}, S_{j}\right)=(\Delta G, \Delta W)$ between two pixels separated by a spatial vector with distance $l$ and orientation $\theta$. When texture is stationary, SDOM is centered at the origin of $\Delta G-$ $\Delta W$ plane in a dense distribution as illustrated in Figure 2.

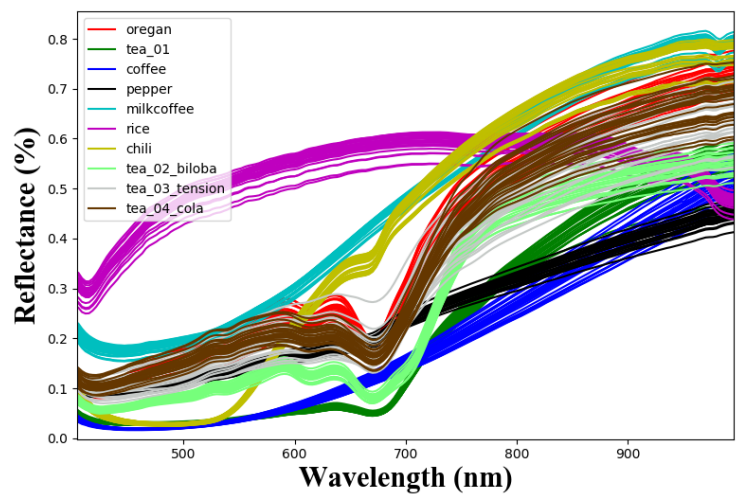

(a) Average spectra (25 for each image)

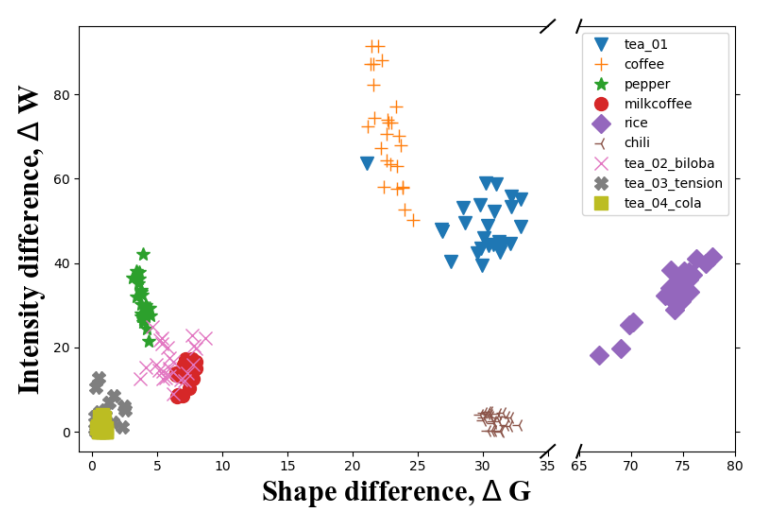

(b) Spectral differences with respect to "Oregon"

Fig. 1: A demonstration of KLPD application with Food images, each split into 25 patches and evaluated independently.

\subsection{Kullback-Leibler divergence as similarity measure}

Considering SDOM as a probability density function, KullbackLeibler (KL) divergence [21] has been identified as the most efficient similarity measure [22]. As the direct calculation of KL divergence is laborious, we proceed to model SDOM using bivariate normal distribution with mean $\mu$ and covariance $\Sigma$. KL divergence can then be estimated as [23]:

$$
\begin{array}{r}
K L\left(M_{1} \| M_{2}\right)=\frac{1}{2}\left[\log \frac{\left|\Sigma_{2}\right|}{\left|\Sigma_{1}\right|}+\operatorname{tr}\left(\Sigma_{2}^{-1} \Sigma_{1}\right)-D\right. \\
\left.+\left(\mu_{2}-\mu_{1}\right)^{T} \Sigma_{2}^{-1}\left(\mu_{2}-\mu_{1}\right)\right]
\end{array}
$$

where $D=2$ is the data dimension. As KL divergence is not symmetric, the SDOM similarity measure is expressed as:

$$
d_{K L}\left(M_{1}, M_{2}\right)=K L\left(M_{1} \| M_{2}\right)+K L\left(M_{2} \| M_{1}\right)
$$




\subsection{Relocated Spectral Difference Occurrence Matrix}

By construction, SDOM is invariant to spectral information as it considers only the spatial variability. For a joint spatialspectral formulation, we modify SDOM into Relocated Spectral Difference Occurrence Matrix (rSDOM) that considers spatial variability around the average spectrum $S_{\mu}$ :

$$
\hat{M}^{(l, \theta)}=\left\{S_{\mu}, M^{(l, \theta)}\right\}
$$

where $S_{\mu}$ is defined over image with $N$ pixels as:

$$
S_{\mu}=\left\{s_{\mu}(\lambda)=\frac{1}{N} \sum_{i=1}^{N} s(\lambda), \forall \lambda \in\left[\lambda_{\min }, \lambda_{\max }\right]\right\}
$$

Essentially, rSDOM is SDOM displaced in the $\Delta G-\Delta W$ plane in accordance to the spectral difference of the average spectrum $d_{K L P D}\left(S_{\mu 1}, S_{\mu 2}\right)=\left(\Delta G_{\mu}, \Delta W_{\mu}\right)$. Consequently, the KL divergence in Equation 7 can be modified as:

$$
\begin{aligned}
K L_{R}\left(\hat{M}_{1} \| \hat{M}_{2}\right)= & \frac{1}{2}\left[\log \frac{\left|\Sigma_{2}\right|}{\left|\Sigma_{1}\right|}+\operatorname{tr}\left(\Sigma_{2}^{-1} \Sigma_{1}\right)-2\right. \\
& \left.+\left[\begin{array}{c}
\Delta G_{\mu} \\
\Delta W_{\mu}
\end{array}\right]^{T} \Sigma_{2}^{-1}\left[\begin{array}{c}
\Delta G_{\mu} \\
\Delta W_{\mu}
\end{array}\right]\right]
\end{aligned}
$$

noting that $\mu$ is close to zero for stationary textures. The rSDOM similarity measure is then expressed as:

$$
d_{K L_{R}}\left(\hat{M}_{1}, \hat{M}_{2}\right)=K L_{R}\left(\hat{M}_{1} \| \hat{M}_{2}\right)+K L_{R}\left(\hat{M}_{2} \| \hat{M}_{1}\right)
$$

We have thus fully defined our proposed hyperspectral texture descriptor rSDOM with its similarity measure in a metrological framework.

\section{EXPERIMENT AND DISCUSSION}

To assess the efficiency of rSDOM, we apply a classification scheme on a hyperspectral texture dataset. We analyze and discuss the performance of rSDOM with reference to Opponent Band Local Binary Pattern (OBLBP). We also list down the limitations of our approach for future improvements.

\subsection{Classification using nearest neighbor}

HyTexiLa [10] is a hyperspectral texture dataset consists of 112 images from five categories: Food (10 images), Stone (4 images), Textile (65 images), Vegetation (15 images) and Wood (18 images). The spectral range is $405.37 \mathrm{~nm}-995.83$ $\mathrm{nm}$, spanning the visible and near infrared (NIR) parts of the electromagnetic spectrum. Each image measures $N=$ $1024 \times 1024$ with $L=186$ spectral bands. The interest of using HyTexiLa is that there exists a complete ground truth

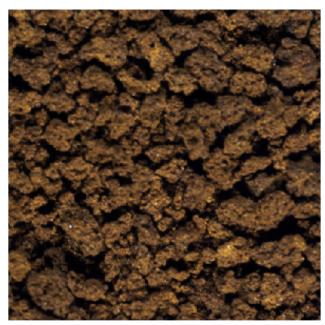

(a) coffee
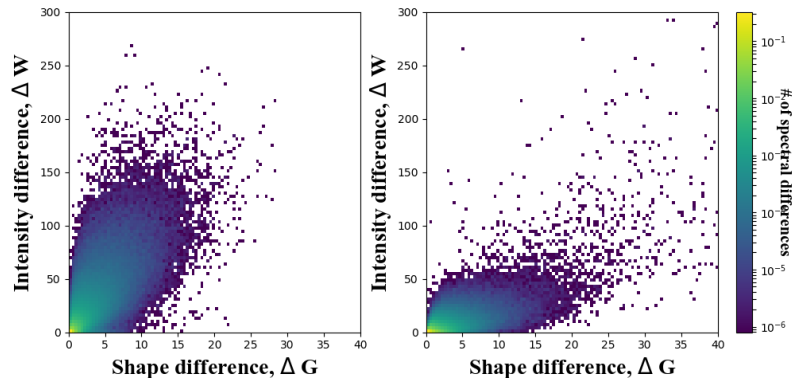

Fig. 2: sRGB-rendered image and Spectral Difference Occurrence Matrix (SDOM) of "Coffee" and "Milkcoffee".

as each pixel is associated with a known texture. This is unlike other datasets such as Pavia University, Indian Pines and Salinas-A for which a part of the pixels is manually labeled.

Following the setting in [10], we split each image into 25 patches, using 12 of them for training and 13 for testing. Considering each image as a class on its own, we perform classification using nearest neighbor which assigns the query image to class in the training set which has the minimum distance with. This is to address the lack of global stationarity that is common in natural scenes, hence improving robustness of the classification scheme. Both intra-categorical and inter-categorical (denoted as $\mathrm{All}$ ) classification are performed using $T=10$ trials with random selection of training and testing sets. The average accuracy and standard error are reported, with the later defined as standard deviation $/ \sqrt{T}$. All calculation is performed using $l=3$ and $\theta=0$.

\subsection{Result and analysis}

Figure 2 depicts the sRGB rendering (only a quarter of the full image is displayed) and SDOM for "Coffee" and "Milkcoffee". Visually, "Coffee" is identified by its coarser structure while "Milkcoffee" exhibits a smoother surface. Clearly, SDOM (and hence rSDOM) is able to capture this texture difference in an easily understandable manner. On shape difference $\Delta G$, both shows identical distribution which is explainable from the fact that they are of the same food origin albeit at different processing level. On intensity difference 
$\Delta W$, "Coffee" is more pronounced than "Milkcoffee" which is expected due to their difference in granular size. Evidently, "Coffee" is grainier and its high surface non-uniformity promotes various kinds of light reflection, inducing different shades of color varying from very dark to very light brownish-like appearance. On the other hand, "Milkcoffee" is powder-like with very fine particle size, making it a perfect light diffuser for uniform surface appearance.

To demonstrate efficiency of the joint spatial-spectral formulation of rSDOM, we present three results in Table 1 that represents classification based on spectral information (average spectrum, A. Spec.), spatial variability (SDOM) and both (rSDOM). It can be observed that rSDOM performs best in all classification (except in Food), with accuracy ranging from 85.9 to $99.7 \%$ which is considered excellent. Expectedly, SDOM registers poorer performance $(47.1-79.9 \%)$ as it disregards spectral information which plays an important role in texture discrimination. On the other hand, it is interesting to note that the performance of average spectrum is quite high (79.0 - 98.7\%). This may be attributed to the fact that HyTexiLa is a relatively small dataset with limited spectral and spatial variety for hyperspectral texture assessment.

From the intra-categorical classification, it can be seen that the rSDOM misclassification in All is mainly due to Vegetation and Wood images. For this, we identify three reasons. Firstly, the texture are predominantly green for Vegetation and brown for Wood, therefore limiting discrimination from each other. Secondly, the choice of $l=3$ prevents rSDOM from assessing texture with larger texton (repeating geometrical structure) size. This explains the better classification of Food, Stone and Textile which consist of mainly fine textons, but reduced performance in classification of Wood and Vegetation with larger texton size. Thirdly, rSDOM is only able to assess texture varying in vertical direction but not in others with the unitary choice of $\theta=0$. This is acceptable for isotropic textures, but not adapted for anisotropic ones such as those found in Vegetation and Wood.

\subsection{Discussion and future work}

In [10], a texture classification scheme is applied on HyTexiLa using Opponent Band Local Binary Pattern (OBLBP). A maximum score of $98.76 \%$ (with 18 principal components) on the inter-categorical classification is reported, while the intra-categorical results are unavailable. In comparison, the performance of rSDOM is slightly lower (94.7\%) although in the same efficiency range. This is possibly due to the fact that rSDOM cannot assess texture directionality. While rSDOM evaluates texture in one direction, OBLBP is able to do so in eight directions, hence the better performance of the later.

Nevertheless, we would like to highlight the fact that rSDOM is extremely lightweight compared to OBLBP. The feature size of OBLBP is $L^{2} \cdot 2^{P}$ for $L$ spectral bands and texture evaluation in $P$ directions. For 18 principal components

\begin{tabular}{|c|c|c|c|}
\hline Category & A. Spec. (\%) & SDOM (\%) & rSDOM (\%) \\
\hline Food & $\mathbf{9 6 . 3} \pm \mathbf{0 . 4}$ & $79.9 \pm 0.7$ & $91.5 \pm 0.6$ \\
\hline Stone & $87.9 \pm 2.2$ & $75.2 \pm 1.5$ & $\mathbf{9 4 . 6} \pm \mathbf{1 . 1}$ \\
\hline Textile & $98.7 \pm 0.1$ & $79.7 \pm 0.4$ & $\mathbf{9 9 . 7} \pm \mathbf{0 . 1}$ \\
\hline Vegetation & $84.7 \pm 0.5$ & $47.1 \pm 0.6$ & $\mathbf{8 8 . 9} \pm \mathbf{0 . 4}$ \\
\hline Wood & $79.0 \pm 0.6$ & $51.4 \pm 0.8$ & $\mathbf{8 5 . 9} \pm \mathbf{0 . 8}$ \\
\hline \hline All & $92.0 \pm 0.2$ & $62.1 \pm 0.3$ & $\mathbf{9 4 . 7} \pm \mathbf{0 . 1}$ \\
\hline
\end{tabular}

Table 1: Classification performance on HyTexiLa dataset.

and texture assessment in 8 directions, OBLBP has a size of $18^{2} \cdot 2^{8}=82944$. In comparison, rSDOM is represented using $D+D^{2}+L=2+4+186=196$ scalar values which is about $0.24 \%$ of OBLBP's, where $D+D^{2}$ is due to the bivariate $(D=2)$ normal approximation. Such significant difference is also reflected in the computational complexity. Considering an image of $N$ pixels, the rSDOM complexity is $O(N \cdot L)$ in contrast to OBLBP's $O\left(N \cdot P \cdot 2^{L}\right)$. For each patch of $N=204 \times 204$ in the HyTexiLa classification scheme, the rSDOM computational time is about two seconds.

One of the limitations in our work lies in the assumption that rSDOM is normally distributed. In fact, this is not exactly the case and statistical transformation may be required to conform better to the normality assumption. On the other hand, Gaussian Mixture Model (GMM) could be used to improve rSDOM modeling for multi-modal distribution of spectral difference. Last but not least, rSDOM would benefit too from a future multi-scale and multi-direction implementation.

\section{CONCLUSION}

We have proposed a new hyperspectral texture descriptor named Relocated Spectral Difference Occurrence Matrix (rSDOM). It assesses the distribution of spectral difference in a given neighborhood around the average spectrum. For spectral distance calculation, rSDOM employs Kullback-Leibler pseudo-divergence (KLPD). Adapted for any spectral range and number of band, it is suitable for industrial and medical applications whereby precision, reproducibility and metrological traceability are of utmost importance. Thanks to its distance-based construction, the curse of dimensionality is solved without requiring any spectral band reduction, therefore preserving all the metrological properties.

The performance of rSDOM has been assessed via a texture classification scheme on HyTexiLa dataset. The obtained results confirms the efficiency of its joint spatial-spectral formulation, with performance exceeding discrimination based on spatial variability or spectral information alone. The performance is also close to Opponent Band Local Binary Pattern (OBLBP), even though rSDOM is processed only for one spatial distance and direction. Besides, rSDOM is extremely lightweight with feature about the size of number of spectral bands, or about $0.24 \%$ of OBLBP's in this work. 


\section{REFERENCES}

[1] A. F. H. Goetz, G. Vane, J. E. Solomon, and B. N. Rock, "Imaging spectrometry for earth remote sensing," Science, vol. 228, no. 4704, pp. 1147-1153, 1985.

[2] N. Pettorelli, J. O. Vik, A. Mysterud, J. Gaillard, C. J. Tucker, and N. C. Stenseth, "Using the satellite-derived ndvi to assess ecological responses to environmental change," Trends in Ecology and Evolution, vol. 20, no. 9, pp. $503-510,2005$.

[3] C. Wang, W. Zheng, Y. Bu, S. Chang, S. Zhang, and R. X. Xu, "Multi-scale hyperspectral imaging of cervical neoplasia," Archives of Gynecology and Obstetrics, vol. 293, no. 6, pp. 1309-1317, 2016.

[4] R. Siche, R. Vejarano, V. Aredo, L. Velasquez, E. Saldaña, and R. Quevedo, "Evaluation of food quality and safety with hyperspectral imaging (HSI)," Food Engineering Review, vol. 8, no. 3, pp. 306-322, 2016.

[5] R. M. Haralick, K. Shanmugam, and I. Dinstein, "Textural feature for image classification," IEEE Trans. on Systems, Man and Cybernetics, vol. 3, no. 6, pp. 610$621,1973$.

[6] Mary M. Galloway, "Texture analysis using gray level run lengths," Computer Graphics and Image Processing, vol. 4, no. 2, pp. $172-179,1975$.

[7] J. Huang, S. R. Kumar, M. Mitra, W. J. Zhu, and R. Zabih, "Image indexing using color correlograms," in Proceedings of IEEE Computer Society Conference on Computer Vision and Pattern Recognition, 1997, pp. $762-768$.

[8] T. Ojala, M. Pietikäinen, and T. Mäenpää, "Multiresolution gray-scale and rotation invariant texture classification with local binary patterns," IEEE Trans. on Pattern Analysis and Machine Intelligence, vol. 24, no. 7, pp. 971-987, 2002.

[9] V. Arvis, C. Debain, M. Berducat, and A. Benassi, "Generalization of the co-occurrence matrix for colour images: Application to colour texture segmentation," Image Anal Estereol, vol. 23, no. I, pp. 63-72, 2004.

[10] H. A. Khan, S. Mihoubi, B. Mathon, J. B. Thomas, and J. Y. Hardeberg, "Hytexila: High resolution visible and near infrared hyperspectral texture images," Sensors, vol. 18 , no. $7,2018$.

[11] Fuan Tsai, Chun-Kai Chang, Jian-Yeo Rau, Tang-Huang Lin, and Gin-Ron Liu, "3d computation of gray level co-occurrence in hyperspectral image cubes," in Energy Minimization Methods in Computer Vision and Pattern Recognition, Alan L. Yuille, Song-Chun Zhu, Daniel
Cremers, and Yongtian Wang, Eds., Berlin, Heidelberg, 2007, pp. 429-440, Springer Berlin Heidelberg.

[12] X. Guo, X. Huang, and L. Zhang, "Three-dimensional wavelet texture feature extraction and classification for multi/hyperspectral imagery," IEEE Geoscience and Remote Sensing Letters, vol. 11, no. 12, pp. 2183-2187, 2014.

[13] H. Zeng and H. J. Trussell, "Dimensionality reduction in hyperspectral image classification," in 2004 International Conference on Image Processing, 2004. ICIP '04, 2004, vol. 2, pp. 913-916.

[14] H. Huang and M. Yang, "Dimensionality reduction of hyperspectral images with sparse discriminant embedding," IEEE Trans. on Geoscience and Remote Sensing, vol. 53, no. 19, 2015.

[15] Y. Voisin S. L. Moan, A. Mansouri and J. Y. Hardeberg, "A constrained band selection method based on information measures for spectral image color visualization," IEEE Trans. on Geoscience and Remote Sensing, vol. 49, no. 12, 2011.

[16] B. Guo, S. R. Gunn, R. I. Damper, and J. D. B. Nelson, "Band selection for hyperspectral image classification using mutual information," IEEE Geoscience and Remote Sensing Letters, vol. 3, no. 4, pp. 522-526, 2006.

[17] H. Deborah, N. Richard, and J. Y. Hardeberg, "A comprehensive evaluation of spectral distance functions and metrics for hyperspectral image processing," IEEE Journal of Sel. Top. in Applied Earth Observations and Remote Sensing, vol. 8, no. 6, pp. 3224-3234, 2015.

[18] N. Richard, D. Helbert, C. Olivier, and M. Tamisier, "Pseudo-Divergence and Bidimensional Histogram of Spectral Differences for Hyperspectral Image Processing," Journal of Imaging Science and Technology, 2016.

[19] B. Julesz, "Visual pattern discrimination," IRE Trans. on Information Theory, vol. 8, no. 2, pp. 84-92, 1962.

[20] M. Unser, "Sum and difference histograms for texture classification," IEEE Trans. on Pattern Analysis and Machine Intelligence, vol. 8, no. 1, pp. 118-125, 1986.

[21] S. Kullback and R. A. Leibler, "On information and sufficiency," The Annals of Mathematical Statistics, vol. 22, no. 1, pp. 79-86, 1951.

[22] Z. Bylinskii, T. Judd, A. Oliva, A. Torralba, and F. Durand, "What do different evaluation metrics tell us about saliency models?," IEEE Trans. on Pattern Analysis and Machine Intelligence, vol. 41, no. 3, pp. 740-757, 2019.

[23] C. E. Rasmussen and C. K. I. Williams, Gaussian Processes for Machine Learning (Adaptive Computation and Machine Learning), The MIT Press, 2005. 